\title{
Emerging role of integrase inhibitors in the management of treatment-experienced patients with HIV infection
}

\author{
Christine Katlama \\ Robert Murphy \\ Pierre et Marie Curie \\ Université - Paris 6, Service de \\ Maladies Infectieuses et Tropicales, \\ Paris, France
}

Correspondence: Christine Katlama Hôpital Pitié-Salpêtrière, Service de Maladies Infectieuses et Tropicales,

47-83 Boulevard de l'Hôpital, 750 I3

Paris, France

Tel +33 I 42 I6 0 I 42

Fax +33 । 42 I6 0130

Email christine.katlama@psl.aphp.fr

\begin{abstract}
Integrase is an essential HIV-1-specific enzyme that is an active target for antiretroviral drug development. Recently, a new class of drugs that specifically inhibits strand transfer, one of the three steps of HIV integration into the host DNA, has been developed. Two drugs in this class have reached late stages of development for use in HIV-1 infected individuals: raltegravir, which has just been approved for use in treatment-experienced patients, and elvitegravir, currently in phase III trials. Both are potent with an $\mathrm{IC}_{50}$ in the $30 \mathrm{nM}$ range and active in vitro against wild type as well as in strains highly resistant to all other existing classes of drugs. Clinical trials in both treatment-naïve and -experienced patients have demonstrated raltegravir to be highly effective with an excellent tolerability profile and no specific clinical or metabolic side effects. Longer follow up is necessary to ensure this early safety profile is sustained. The rapid rate of viral decay observed with raltegravir challenges the current understanding of HIV-1 turnover and may open new strategies for long term treatment and management of infected patients.
\end{abstract}

Keywords: integrase inhibitors, antiretroviral therapy, treatment failure, raltegravir, elvitegravir

\section{Background}

Current estimates suggest that there are 33.2 million persons living with HIV infection and that despite ongoing prevention efforts, 2.5 million new infections occurred in 2007 as the pandemic continues. The year 2007 was, however, remarkable on many fronts: prevalence rates globally appeared to stabilize or decrease slightly; the total number of HIV-infected individuals who were receiving antiviral therapy increased to approximately 2.6 million persons, the majority now being in resource-limited settings; and new antiretroviral drugs continued to be developed including two from new classes which received approval for treatment in HIV-infected patients. ${ }^{1,2}$

There were setbacks in 2007 as well, mostly on the prevention front. The biggest disappointment was the failure of the adenovirus vector vaccine trials, the results of which hinted that vaccine recipients may have actually done worse that those who received placebo. ${ }^{3,4}$ Similar failures were observed in microbicide prevention trials. ${ }^{5,6}$ Additionally, it was conclusively demonstrated that interrupting therapy was actually harmful, ending that strategy as a viable alternative. ${ }^{7}$ The focus on antiretroviral treatment, therefore, has retaken center stage. Treatment of HIV-infection with antiretroviral agents has regained its importance for multiple reasons: the recent interventional prevention trials have failed, treatment in the developing world has been very rapid and successful, and the newer generation of antiretroviral drugs recently approved or in development are very potent and offer significant advantages in tolerance and efficacy compared to many of the existing drugs. 
For the past 12 years, there have been only 2 HIV targets where therapy has been successful in blocking viral replication, reverse transcriptase and protease. Although there is a drug that inhibits fusion, to date this treatment is considered difficult and is infrequently utilized primarily because of the parenteral administration involved. We now have additional options with drugs from 2 new classes: inhibitors of viral integrase and small molecules capable of blocking the CCR5 chemokine receptor which is essential for viral entry into the cell.

One of the most exciting areas in HIV treatment and research today involves the integrase inhibitors. Integrase is an HIV viral enzyme that is essential for viral replication. Inhibitors of integrase are completely independent in their activity compared to all other antiretroviral drug classes including reverse transcriptase, protease, maturation and entry inhibitors. Virus resistant to all know drug classes therefore, remain completely susceptible to inhibitors of integrase. Additionally, integrase inhibitors are active in both CCR5-tropic and CXCR4-tropic HIV-1 viruses. ${ }^{8,9}$

One of the first integrase inhibitors to be developed is raltegravir (MK-0518). This drug has been shown to have a very potent and rapid antiretroviral effect with a $2 \log$ reduction in plasma HIV RNA in 10 days of monotherapy. ${ }^{10}$ This drug recently received accelerated but conditional approval by the United States Food and Drug Administration and the European Medicines Evaluations Agency for the treatment of patients failing existing therapy. Further development for use in treatment-naïve patients is ongoing. The second integrase inhibitor to be developed is elvitegravir (GS 9137). This review focuses on these 2 new drugs that inhibit HIV-1 integrase. Mechanism of action, pharmacokinetics, clinical use in treatment-experienced patients, clinical use in treatment-naïve patients, tolerability, resistance profile, and other potential uses are addressed. The chemical structures of these 2 compounds are illustrated in Figure 1.

\section{Mechanism of action for integrase inhibition}

Integrase is an HIV-1 enzyme that is essential for viral replication. Integrase catalyzes at least 3 reactions: 3 ' processing, formation of the preintegrase complex, and strand transfer (Figure 2). Briefly, once the viral RNA is retrotranscribed into DNA by reverse transcriptase, integrase removes a 3' terminal portion at both ends of the newly formed DNA where it remains bound, stabilizing the preintegrase complex. Once 3' processing occurs, the preintegrase complex
A
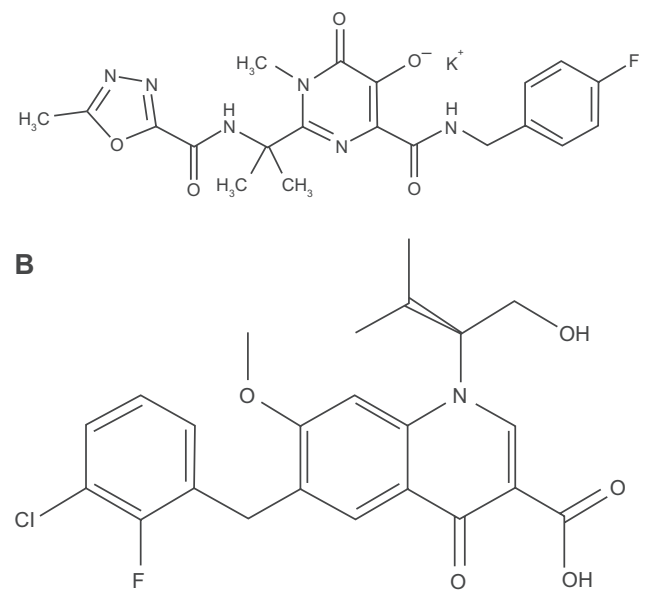

Figure I Chemical structure of 2 integrase inhibitors: a) raltegravir - a diketoacid and b) elvitegravir - a hydroxyquinolone.

can form which consists of ring-shaped viral DNA with associated virus and host proteins. This complex structure is able to translocate across the nuclear membrane and into the nucleus. The final reaction catalyzed by integrase is the strand transfer where both 3' ends of the DNA are inserted into the host DNA or chromosome. The newly altered host DNA, which now includes the HIV DNA, requires repair which is done with cellular DNA repair enzymes. If strand transfer in inhibited, the viral ring structures remain in the nuclear cytoplasm, the clinical relevance of which is unknown. ${ }^{11-12}$ Inhibitors of integrase that have now been developed are strand transfer inhibitors (INSTIs) that block the insertion position on the host DNA. Three integrase inhibitor classes of compounds have been identified: diketoacids, hydroxyquinolones, and polyphenols. Raltegravir is a member of the diketoacid class and elvitegravir belongs to the hydroxyquinolone class. ${ }^{14}$

In preclinical studies, raltegravir was found to be effective in vitro and safe in animal models. In HIV-1 isolates including 6 subtypes with both syncytium and non-syncytium inducing isolates, raltegravir inhibited replication in peripheral blood mononuclear cells (PBMCs) with cellular $\mathrm{IC}_{95}$ values ranging from 6 to $50 \mathrm{nM}$. It also inhibited replication in laboratory HIV-2 isolates, with an average cell $\mathrm{IC}_{95}$ of $6.3 \mathrm{nM}$. Raltegravir did not inhibit any human DNA polymerases significantly nor did it inhibit any tested enzyme activity, transporter or receptor-ligand assays. The toxicologic effects of raltegravir in the dog and rat were not significant, carcinogenicity studies have been negative to date, and the reproduction toxicity studies in rats and rabbits were unremarkable; hence raltegravir has been classified as a Pregnancy Category C Drug. ${ }^{15-17}$ 


\section{Integrase Presents Multiple Potential \\ Targets for Intervention}

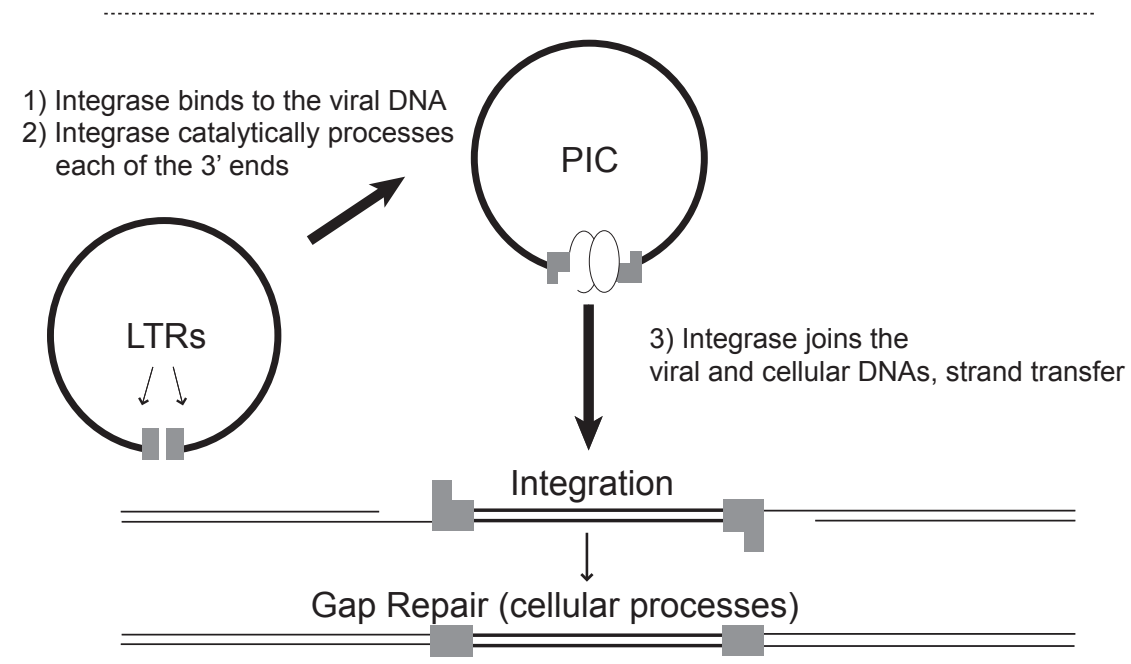

Figure 2 Integrase: mechanism of action for integrase inhibition. Modified from Hazuda DJ. 2006. Inhibitors of human immunodeficiency virus type I integration. Curr Opin HIV AIDS. I:212-217.36

Abbreviations: PIC, preintegration complex; LTRs, long terminal repeats.

\section{Pharmacokinetics of integrase inhibitors}

One of the main differences between the two most advanced INSTI drugs in development is their pharmacokinetic properties.

\section{Raltegravir}

Raltegravir is primarily metabolized by glucuronidation and is mainly excreted in faeces (51\%) with the remainder excreted in urine $(32 \%)$. In humans, there is only one metabolite, M2, which accounts for most of the dose recovered in urine. It is neither a substrate for nor an inhibitor of cytochrome $\mathrm{P} 450$ enzymes; therefore, it is not expected to have significant drug-drug interactions with drugs that affect or are influenced by $\mathrm{P} 450$ isoenzymes. Raltegravir is $83 \%$ bound to human proteins in plasma. The half-life of raltegravir is approximately 9 hours. Twelve hours after dosing, the concentration in humans exceeded $33 \mathrm{nM}$ (the in vitro $\mathrm{IC}_{95}$ for HIV-1 in 50\% normal serum) in all treatment groups tested, thus confirming the twice daily dosing schedule that has been approved for use. The recommended dose is $400 \mathrm{mg}$ twice daily administered without regard to fasting and the drug is supplied in a $400 \mathrm{mg}$ pill formulation.

Age, gender, race, food, hepatic impairment (Child-Pugh scores 7-8) and renal dysfunction had no significant effects on raltegravir pharmacokinetics. Raltegravir has not be studied in patients with severe hepatic impairment, but no significant impact on drug levels is expected; nor has it been studied in patients on dialysis and therefore it should be avoided in that clinical situation, but if necessary, therapeutic drug monitoring should be considered. Typical peak plasma concentrations in uninfected adults are $4.5 \mu \mathrm{mol} / \mathrm{L}$ and concentrations after 12 hours are $142 \mathrm{nmol} / \mathrm{L} \cdot{ }^{17-20}$ Raltegravir does not readily cross the blood-brain barrier (Gilles Peytavin, personal communication).

Because raltegravir is mainly metabolized through the liver enzyme uridine diphosphate-glucuronosyl-transferase $1 \mathrm{~A} 1$ (UGT1A1), concentrations are increased by $53 \%$ if co-administered with atazanavir, a drug which affects glucuronidation; however this was associated with no increase in adverse effects and no dosing adjustments are recommended..$^{21,22}$ Similarly, co-administration with drugs that induce UGT1A1, like rifampin, phenytoin and phenobarbital, should be done cautiously and avoided if at all possible. If rifampin must be co-administered, consideration should be given to doubling the dose of raltegravir as recommended in the European Union guidelines; however this approach is not recommended by regulatory authorities in the United States or by the manufacturer. ${ }^{17}$

One of the pharmacologic advantages of raltegravir is its lack of significant interactions with drugs metabolized by cytochrome P450 isoenzymes. Consequently, there are no significant drug interactions noted nor dosing adjustments required with midazolam, efavirenz, ritonavir or any of the protease inhibitors, including tipranavir. ${ }^{17,23}$ 


\section{Elvitegravir}

Elvitegravir is a modified quinolone antibiotic that is also a potent INSTI. Elvitegravir undergoes both oxidative metabolism by CYP3A4 and glucuronidation. It is a moderate inducer of CYP3A4 and significant drug-drug interactions may occur when other CYP3A4 inducers or inhibitors are concomitantly administered. In healthy volunteers, the plasma half-life was approximately 3 hours when the drug was given alone but half-life is significantly enhanced if an inhibitor of CYP3A4, such as ritonavir, is co-administered. When ritonavir is co-administered, steady state exposure and minimum plasma concentrations increase 20-fold and 90-fold, respectively and the plasma half-life increases to approximately 9 hours, which then potentially allows for once daily dosing (Figure 3). Absorption of elvitegravir is increased approximately 3-fold when the drug is co-administered with food. Because of the superior performance of the highest dose tested in the phase II trial, the drug is being developed as $150 \mathrm{mg}$ twice daily administration with ritonavir $100 \mathrm{mg}$ in treatment-experienced patients. ${ }^{24,25}$

\section{Clinical use in treatment-experienced patients}

Just as many of the more recent antiretroviral agents have developed, the initial clinical development for these integrase inhibitors has focused on the treatment-experienced HIV-infected patient population with drug resistance to the available classes of antiretroviral agents.

\section{Raltegravir}

A total of 878 patients were studied in the treatment-experienced trials of raltegravir: one phase IIb clinical study and 2 large identical phase III studies performed in HIV-infected patients with triple class drug resistance.

The first study was a phase II dose-ranging study in 179 treatment-experienced patients with plasma HIV RNA $>5000$ copies/mL who were receiving stable antiretroviral treatment (ART) for at least 3 months with documented resistance to at least one drug in each of the 3 classes of ART. They were randomized to optimized therapy (OT) or OT plus $200 \mathrm{mg}$, $400 \mathrm{mg}$ or $600 \mathrm{mg}$ twice daily raltegravir. Prior to randomization, optimized therapy was selected based on antiretroviral history, resistance testing, and prior clinical or laboratory toxicities. This population was highly treatment-experienced and had received ART for approximately 10 years prior to study entry. The baseline plasma HIV RNA level was $4.7 \log$ copies/mL and median CD4 count was 240 cells $/ \mathrm{mm}^{3}$. Patients had a very high level of drug resistance: $66 \%$ had a genotype sensitivity score (GSS) of $0,98 \%$ were not susceptible to any approved PI, and $36 \%$ were taking the fusion inhibitor, enfuvirtide, in their background therapy.

\section{Gs study $183 \quad$ Elvitegravir (GS 9137) Steady-State Pharmacokinetics}

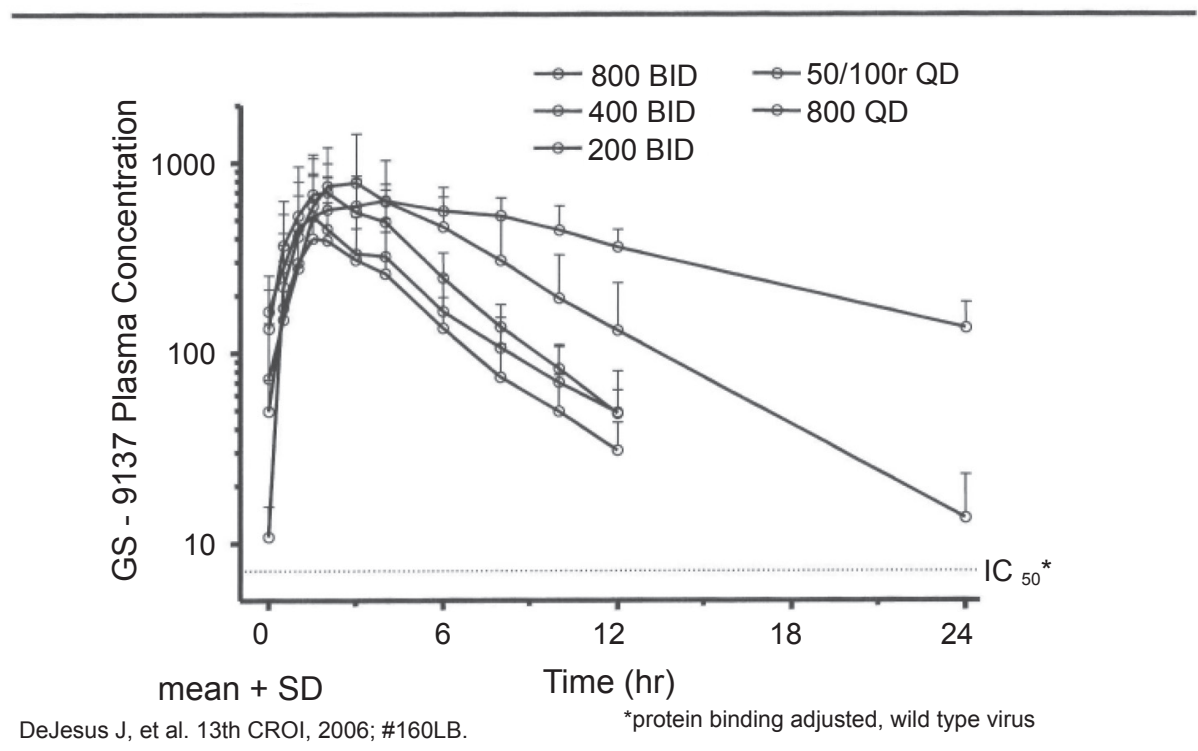

Figure 3 Pharmacokinetic properties of elvitegravir with and without ritonavir. Modified with permission from Iwamoto M, Kassahun K, Troyer MD, et al 2008. Lack of pharmacokinetic effect of raltegravir on midazolam: in vitro/in vivo correlation.J Clin Pharmacol. 48(2):209-214. 
In all the raltegravir groups, there was an approximate $2.0 \log _{10}$ drop in plasma HIV RNA by week 24, compared to optimized therapy alone with placebo which decreased by only $0.35 \log _{10}$ copies $/ \mathrm{mL}(\mathrm{p}<0.0001)$. The proportion of patients who decreased their HIV RNA to $<400$ copies $/ \mathrm{mL}$ at week 24 were $69.8 \%, 71.1 \%$ and $71.1 \%$ in the $200 \mathrm{mg}$, $400 \mathrm{mg}$ and $600 \mathrm{mg}$ raltergravir arms, respectively, compared to $12 \%$ in the OT group $(\mathrm{p}<0.0001)$. The proportion of patients with HIV RNA $<50$ copies $/ \mathrm{mL}$ at week 24 was $65.1 \%, 55.6 \%$ and $66.7 \%$ in the raltegravir $200 \mathrm{mg}, 400 \mathrm{mg}$ and $600 \mathrm{mg}$ arms, respectively, compared to $13.3 \%$ in the placebo arm $(\mathrm{p}<0.0001)$. There were no significant difference in viral efficacy between the 3 dosage groups studied. The CD 4 counts increase by 51 to 94 cells $/ \mathrm{mm}^{3}$ in the raltegravir groups compared to a decrease of $16 \mathrm{cells} / \mathrm{mm}^{3}$ in the placebo group $(\mathrm{p}<0.0001)$. The use of enfuvirtide in the OT improved viral outcomes in all groups. There were few adverse events that led to treatment discontinuation. Based on these findings, and those from a phase II treatment-naïve study, the $400 \mathrm{mg}$ twice daily dose was chosen for use in the phase III trials. ${ }^{22}$

Two double-blind phase III studies with identical protocols were performed. BENCHMRK 1 was conducted at 65 sites in Europe, Asia and South America and BENCHMRK 2 was conducted at 53 sites in North and South America. Patients enrolled into these trials had plasma HIV RNA $>1000$ copies $/ \mathrm{mL}$ and were on stable ART for at least 2 months. They had to have reduced susceptibility to at least one drug in each of the existing 3 classes of ART defined as nonnucleoside reverse transcriptase inhibitors (NNRTIs), nucleoside analog reverse transcriptase inhibitors (NRTIs) and protease inhibitors (PIs). Patients were randomized to receive raltegravir $400 \mathrm{mg}$ twice daily or matching placebo taken without regard to food. Background therapy could include investigational drugs that were under review for licensure. Here, for the first time in a treatment-experienced trial, the primary endpoint was the proportion of patients with "undetectable" HIV RNA at week 16 with a cutoff of $<400$ copies $/ \mathrm{mL}$. Patients were stratified by enfuvirtide use and whether they had resistance to 1 or $>1$ PI. After week 16 , patients with virologic failure were allowed to receive openlabel raltegravir.

Since the BENCHMRK 1 and 2 studies were identical, the combined results are reported. Overall, 699 patients were enrolled. Patients were highly treatment experienced with $>90 \%$ having a history of AIDS and a median 10 years of prior ART with 12 different drugs. The median CD4 count was 119 cells $/ \mathrm{mm}^{3}$ and 123 cells $/ \mathrm{mm}^{3}$ and the median plasma HIV RNA was $4.8 \log _{10}$ copies/mL and $4.7 \log _{10}$ copies $/ \mathrm{mL}$ for the raltegravir-treated and placebo patients, respectively. Overall, $96.3 \%$ of patients were resistant to $>1$ PI. Two thirds of patients had a GSS of 0 or 1 . The proportion who used enfuvirtide in their background therapy was approximately $38 \%, 20 \%$ initiating it for the first time in this study.

At week 16, the proportion of patients with HIV RNA $<400$ copies $/ \mathrm{mL}$ was $77.5 \%$ for the raltegravir-treated patients and $41.9 \%$ in the placebo arms $(\mathrm{p}<0.001)$. Suppression to $<50$ copies $/ \mathrm{mL}$ at week 16 was $61.8 \%$ in the raltegravir patients versus $34.7 \%$ in the placebo arms ( $\mathrm{p}<0.001)$. The decrease in plasma HIV RNA at week 24 was $1.8 \log _{10}$ copies $/ \mathrm{mL}$ in the raltegravir arms compared to the $0.9 \log _{10}$ copies $/ \mathrm{mL}$ for the placebo arm $(\mathrm{p}<0.001)$. The mean increase in CD4 count from baseline was 84 cells $/ \mathrm{mm}^{3}$ in the raltegravir patients and $36 \%$ for those receiving placebo $(p<0.001)$ (Figure 4). The treatment effect of raltegravir was consistent regardless of baseline HIV RNA, CD4 cell count, GSS, phenotypic sensitivity score (PSS), inclusion of darunavir and/or enfuvirtide, gender, race, geographic region and viral subtype. In patients using either darunavir or enfuvirtide for the first time, the proportion with HIV RNA $<50$ copies $/ \mathrm{mL}$ at week 24 were $68 \%$ to $82 \%$ in the raltegravir arms compared to $48 \%$ to $50 \%$ in the placebo arms.

The results in the combined analysis were sustained out to 48 weeks: the proportion of patients with HIV RNA $<400$ copies $/ \mathrm{mL}$ was $72.3 \%$ for raltegravir-treated patients compared to $37.1 \%$ for those on placebo. The proportion of patients with HIV RNA $<50$ copies $/ \mathrm{mL}$ was $62.1 \%$ versus $32.9 \%$ in the raltegravir and placebo groups ( $\mathrm{p}<0.001)$. The CD 4 count increased by 109 cells $/ \mathrm{mm}^{3}$ versus 45 cells $/ \mathrm{mm}^{3}$, respectively $(\mathrm{p}<0.001)$. $^{26,27}$

The prognostic factors (odds ratio) predicting $<400$ copies/mL at week 24 included baseline HIV RNA (0.35), enfuvirtide use in enfuvirtide-naïve patients (5.05), active PI in background ART (2.34), and darunavir use (5.78) (Table 1). Discontinuation of treatment because of clinical adverse events was $1.9 \%$ in the raltegravir arms compared to $2.1 \%$ in the control groups. The discontinuation of treatment because of laboratory adverse experiences were $0.2 \%$ in the raltegravir arms compared to $0.0 \%$ in the control arms. ${ }^{28}$

\section{Elvitegravir}

The largest study to date of elvitegravir is a 278 subject phase II, dose ranging, non-inferiority, partially blinded trial in treatment experienced patients with at least 1 PI mutation. 
A

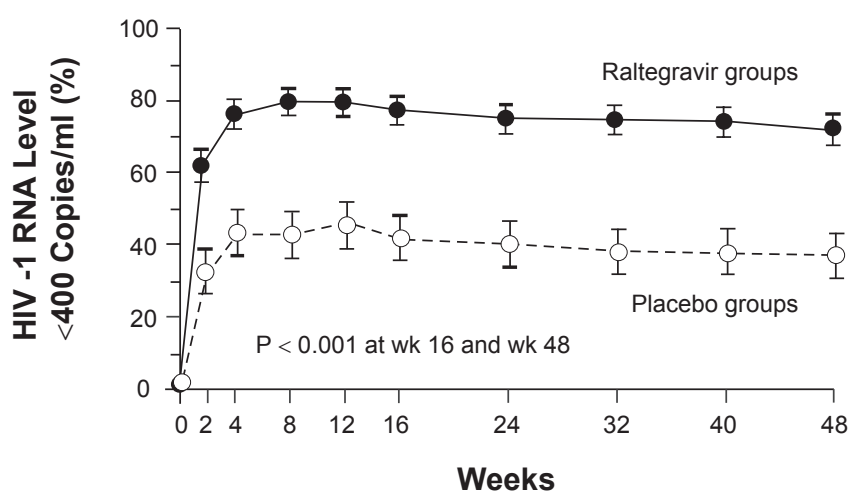

B

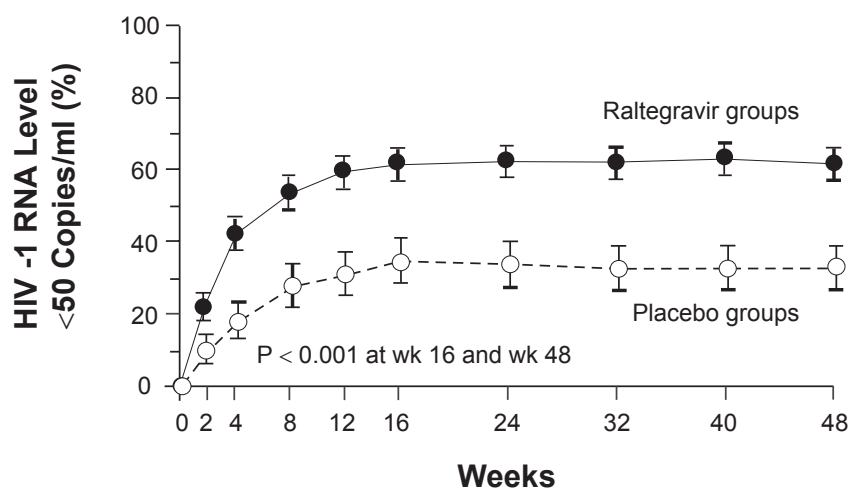

No. of

patients

$\begin{array}{llllllll}\text { Raltegravir } & 462 & 458 & 458 & 461 & 453 & 458 & 459\end{array}$

$\begin{array}{llllllll}\text { Placebo } & 237 & 236 & 236 & 237 & 237 & 237 & 237\end{array}$

Figure 4 BENCHMRK I and 2 combined results at week 48: a) proportion of patients with HIV RNA < 400 copies/ml, and b) proportion of patients with HIV RNA <50 copies/ml. Reproduced with permission from Steigbigel RT, Cooper DA, Kumar PN, et al 2008. Raltegravir with optimized background therapy for resistant HIV infection. N Engl J Med. 359:339-354. ${ }^{27}$ Copyright (C) 2008. Massachusetts Medical Society. All rights reserved.

No NNRTIs were allowed due to lack of knowledge of potential drug-drug interactions at the time the study was designed. Three different doses of elvitegravir were studied: $20 \mathrm{mg} /$ day, $50 \mathrm{mg} /$ day and $125 \mathrm{mg}$ /day. All patients received a boosted PI that included $100 \mathrm{mg}$ of ritonavir in their background regimen. The baseline HIV RNA was $4.54 \log _{10}$ copies $/ \mathrm{mL}$ and the median CD4 counts was 158 cells $/ \mathrm{mm}^{3}$. Patients were highly treatment-experienced and had a median of 11 PI mutations. At week 8 , the elvitegravir $20 \mathrm{mg} /$ day arm was discontinued because of a high rate of virological failure and patients were allowed to switch to the $125 \mathrm{mg} /$ day arm. At week 16,37\% of the control arm patients switched to elvitegravir. At week 24, the time of the primary endpoint, the time-weighted average change from baseline in HIV RNA was $-1.2,-1.5$ and $-1.7 \log _{10}$ copies $/ \mathrm{mL}$ for the control arm, elvitegravir $50 \mathrm{mg} / \mathrm{mL}$ and $125 \mathrm{mg} / \mathrm{mL}$, respectively. The only significant difference between the 3 arms was that the elvitegravir $125 \mathrm{mg} / \mathrm{mL}$ arm was superior to the control PI arm $(p=0.01)$. The proportion of patients with
HIV RNA $<50$ copies/mL at week 16 was $30 \%, 38 \%$ and $40 \%$ for the control, elvitegravir $50 \mathrm{mg}$ /day and elvitegravir $125 \mathrm{mg} /$ day, respectively. The mean increase in CD4 counts was 28,52 and 61 cells $/ \mathrm{mm}^{3}$ in the 3 groups, respectively. ${ }^{29}$ Because these findings demonstrated superior virologic efficacy with the highest drug exposure, further development in treatment-experienced patients will utilize elvitegravir at $150 \mathrm{mg}$ twice daily plus ritonavir $100 \mathrm{mg}$.

\section{Clinical use in treatment-naïve patients \\ Raltegravir}

The only clinical study in treatment-naïve patients is a phase II trial, Study 004. This study was a multi-center, double-blind, randomized dose-ranging study in ART-naïve patients. Part I of this trial included 10 days of raltegravir monotherapy $100 \mathrm{mg}, 200 \mathrm{mg}, 400 \mathrm{mg}$, and $600 \mathrm{mg}$ twice daily $(\mathrm{N}=35) .^{10}$ Following this, Part II of the study combined these doses of raltegravir with standard dosages of tenofovir plus lamivudine 
Table I Odds ratio associated with virologic success in the BENCHMRK I and 2 trials

\begin{tabular}{|c|c|c|c|}
\hline \multirow[t]{2}{*}{ Prognostic factor } & \multicolumn{3}{|c|}{ Odds ratio for prognostic factor* } \\
\hline & Odds ratio & $95 \% \mathrm{Cl}$ & P-value \\
\hline Baseline HIV RNA ( $\log _{10}$ copies/mL) & 0.35 & $(0.26,0.47)$ & $<0.001$ \\
\hline $\begin{array}{l}\text { Enfuvirtide use in OBT in } \\
\text { enfuvirtide-patients (Yes:No) }\end{array}$ & 5.05 & $(2.72,9.38)$ & $<0.001$ \\
\hline $\begin{array}{l}\text { Active } \mathrm{Pl} \text { in OBT determined by } \\
\text { phenotypic resistance test** (Yes:No) }\end{array}$ & 2.34 & $(1.43,3.81)$ & $<0.001$ \\
\hline $\begin{array}{l}\text { Darunavir use in OBT in } \\
\text { Darunavir-naive patients (Yes:No) }\end{array}$ & 5.78 & $(3.55,9.42)$ & $<0.001$ \\
\hline Treatment & 9.24 & $(5.94,14.37)$ & $<0.001)$ \\
\hline
\end{tabular}

Reproduced with permission from Steigbigel RT, Cooper DA, Kumar PN, et al 2008. Raltegravir with optimized background therapy for resistant HIV infection. N Engl J Med. 359:339-354..$^{27}$ Copyright @ 2008. Massachusetts Medical Society. All rights reserved.

*An odds ratio of $(<I,-I,>I)$ indicates (decreased, equal, increased) probability to respond at Week 24. Odds ratio and p-value were calculated using a logistic regression model adjusted for baseline HIV RNA level, enfuvirtide use in OBT with no prior exposure; active PI in OBT determined by phenotypic resistance test, investigational ART use in OBT (Darunavir or tipranavir use), study, and treatment.

**Phenotypic resistance test are not available for enfuvirtide and Darunavir, both of them are excluded.

Abbreviations: OBT,optimal background therapy; P, protease inhibitor;ART, antiretroviral therapy.

and compared this to efavirenz plus tenofovir/lamivudine $(\mathrm{N}=171)$. In Part II, the baseline mean plasma HIV RNA ranged from 4.7 to $4.9 \log _{10}$ copies $/ \mathrm{mL}$ and $\mathrm{CD} 4$ ranged from 225 to 277 cells $/ \mathrm{mm}^{3}$. In Part I, the proportion with HIV RNA $<400$ copies $/ \mathrm{mL}$ at day 10 ranged from $50.0 \%$ to $57.1 \%$ compared to placebo at $0 \%$. In Part II, the proportion with HIV RNA $<400$ copies $/ \mathrm{mL}$ at week 48 was $85.3 \%$ to $100.0 \%$ in the various groups with no significant difference between raltegravir-treated and efavirenz-treated patients. At week $48, \mathrm{CD} 4$ counts increased by 140 to 216 cells $/ \mathrm{mm}^{3}$ in all the groups. Raltegravir was well tolerated in all the treatment arms. Interestingly, the rate of viral decay during treatment was much greater in the raltegravir arms compared to the efavirenz arm. The implications of this finding at the present time are speculative but under intense investigation. ${ }^{20}$

\section{Elvitegravir}

Elvitegravir has not been extensively studied in antiretroviral-naïve patients. There has been a 10 day monotherapy, randomized, double-blind, dose-ranging study in treatmentnaïve and experienced patients. Patients were randomized to receive elvitegravir $800 \mathrm{mg}$ twice daily, $400 \mathrm{mg}$ twice daily, $200 \mathrm{mg}$ twice daily or $800 \mathrm{mg}$ once daily or $50 \mathrm{mg}$ boosted by ritonavir $100 \mathrm{mg}$ daily. The median baseline CD4 count was 442 cell $/ \mathrm{mm}^{3}$ and HIV RNA was $4.75 \log _{10}$ copies $/ \mathrm{mL}$. The mean reduction in HIV RNA was $1.91 \log _{10}$ copies $/ \mathrm{mL}$ in the elvitegravir $800 \mathrm{mg}$ twice daily, $400 \mathrm{mg}$ twice daily and $50 \mathrm{mg}$ daily plus ritonavir. This study demonstrated that the pharmacokinetic boosting by ritonavir may allow for once daily administration in certain populations with a reduced dose of elvitegravir. ${ }^{24}$ Because of concerns by regulators that low dose ritonavir administration in treatment-naïve patients who are not receiving another protease inhibitor may be at risk for developing protease mutations, development of elvitegravir boosted by ritonavir in this population has been delayed pending results of safety and further proof-of-concept studies.

\section{Tolerability and safety Raltegravir}

In the large phase III BENCHMRK trials, the most commonly reported adverse events were diarrhea, nausea and headache. Across all studies, there was an increased incidence of rash in the raltegravir arms, $6.7 \%$ compared to $3.9 \%$ in controls. When raltegravir is co-administered with atazanavir the rate is higher $(9.4 \%)$. With darunavir, the rash rate was $13.5 \%$ for the cumulative period on study compared to $4.0 \%$ in the control group also receiving darunavir. Rash is typically mild to moderate and rarely results in study drug discontinuation. Rash is included as a specific issue in the overall risk management plan for raltegravir.

In the pooled analysis across all the studies, there were no drug-related clinical adverse events that occurred at frequencies at least $2 \%$ greater in the raltegravir groups compared to placebo, however when additional safety information was included, clinical events that were $>2 \%$ in the raltegravir group included fatigue ( $7.9 \%$ versus $4.6 \%$ ), herpes zoster $(4.1 \%$ versus $0.7 \%)$ nasopharyngitis ( $6.1 \%$ versus $3.9 \%)$ and rash $(5.3 \%$ versus $2.5 \%)$.

The tolerability profile of raltegravir is very similar in patients with and without co-infection with hepatitis $\mathrm{B}$ and/or C. 


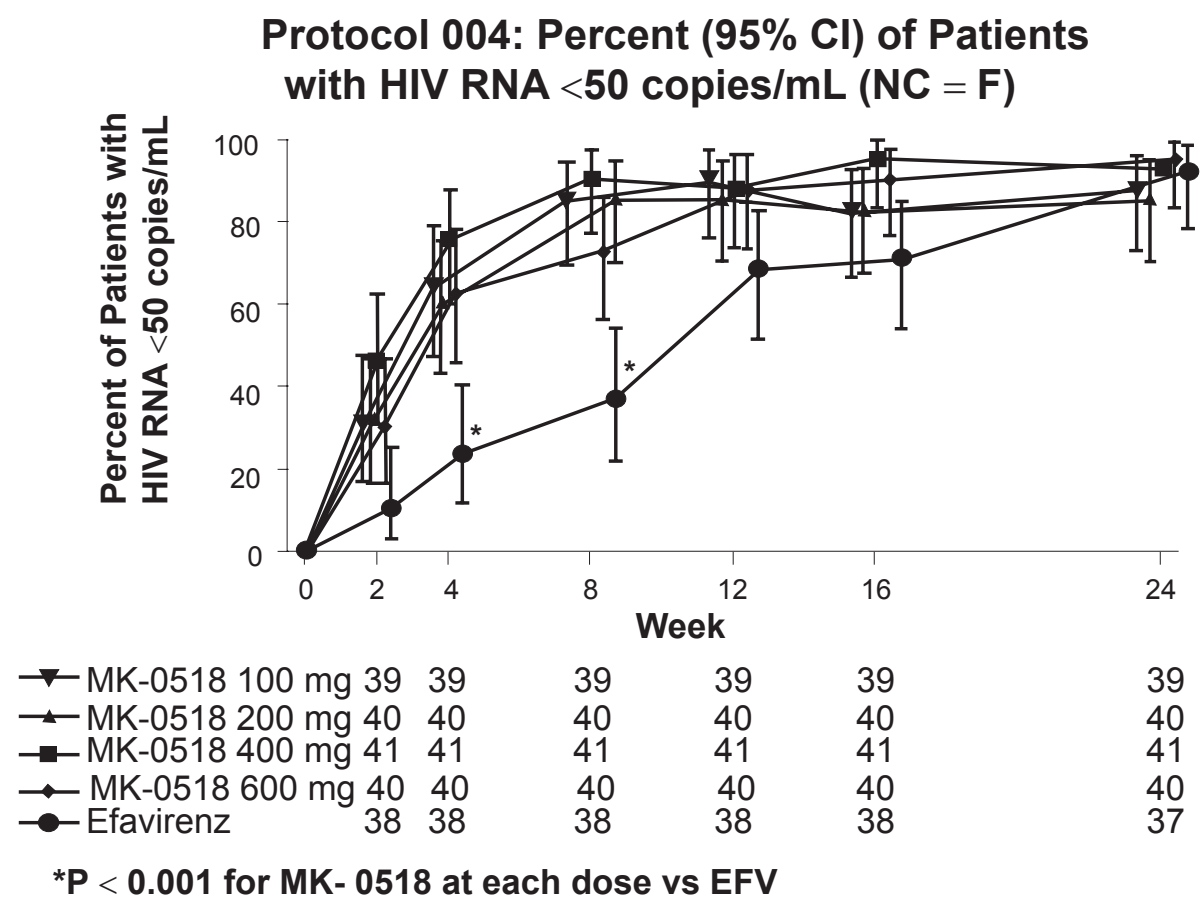

Figure 5 Early virologic effect of raltegravir-based treatment compared to standard efavirenz-based treatment in patients initiating antiretroviral therapy. Modified with permission from McColl DJ, Fransen S, Gupta S, et al 2007. Resistance and cross-resistance to first generation integrase inhibitors: insights from a phase 2 study of elvitegravir (GS-9|37). Antiviral Ther. 12:SII. Abstract 9.33

An imbalance in malignancy rates was originally observed in the BENCHMRK trials but this imbalance was not maintained after 7 months of follow up and no longer considered to be of significance. Cancers were detected in $3.5 \%$ of raltegravir and $1.7 \%$ of placebo recipients. The relative risk of cancer in the raltegravir groups as compared to placebo was 1.54 (95\% CI, 0.50-634).

There appears to be no significant effect of raltegravir on serum lipids. Other laboratory parameters appear not be significantly affected although there are more grade 2 AST and ALT elevations in raltegravir-treated patients compared to placebo. There were very few grade 3 elevations, however. ${ }^{26,28}$

\section{Elvitegravir}

Elvitegravir has been well tolerated in the limited number of studies completed to date. Very few patients have experienced any severe clinically relevant adverse effects. In the phase II trial $1 \%$ to $3 \%$ of patients discontinued treatment because of adverse events. ${ }^{24,29}$

\section{Resistance profile}

In vitro resistance testing was done by culturing laboratory HIV-1 isolate in human $\mathrm{H} 9$ cells in increasing raltegravir concentrations over a period of months. The first change observed was Q148K. Subsequently, E138A and G140A were isolated. The Q148K, E138A/Q148K and E138A/G140A/Q148K mutations resulted in average fold-shift values in $\mathrm{IC}_{50}$ of 46-fold, 90-fold, and 508-fold, respectively. ${ }^{26,30,31}$

In the BENCHMRK trials, by week 24, 84 raltegravirtreated patients had met the definition of virologic failure and integrase genotype data were available for 60 patients. Of these, 42/60 (70\%) showed genotypic evidence of raltegravir resistance. This high proportion of patients with virlogic failure and resistance mutations suggests a relatively low genetic barrier for the development of resistance if viral suppression is not optimal. The risk of developing treatment-emergent mutations was greater in patients with higher baseline HIV RNA levels or GSS or PSS of 0. Virologic failure was generally associated with mutations at either amino acid 148 (Q changed to H, K, Or R) or 155 ( $N$ changed to $\mathrm{H}$ ) and possibly through one other pathway, Y143R/C. The majority $33 / 42(79 \%)$ of viruses at the time of virological failure contained two or more resistance-associated mutations in integrase. Additional mutations associated with the 148 pathway included G140 S/A and E138K; for the 155 pathway, mutations included E92A, V151I, T97A, G163R and L74M; for the 143 pathway, mutations included L74A/I, E92/Q, T97A, I203M and S230R. In a small number, there were 2 or more secondary mutations without a mutation at 
position 148 or 155 . These included mutations at L74, E92, T97, F121, E138, and G140 all of which had a minimal effect on susceptibility. ${ }^{26}$

In $28 / 39$ patients treated with elvitegravir in a phase II study, mutations occurred at E92Q, E138K, Q148R/K/H or $\mathrm{N} 155 \mathrm{H}$ in $11 / 28$ samples In patients who had failed treatment. The presence of the mutations at 148 and 155 positions suggests that elvitegravir and raltegravir are cross resistant. $^{29,32,33}$

\section{Other potential indications}

One of the more exciting features of integrase inihibitor development is the dramatic difference in early phase viral decay in the treatment-naïve patients evaluated in the Raltegravir 004 Study. While the effect at week 24 was basically the same as with a conventional efavirenz-based approach, the earlier declines in HIV RNA were significant when compared to the standard approach. ${ }^{20,34}$ The reason for this is unknown but may be due to the more complete inhibition of integration offered by this category of compounds.

Exploring what can be a further added value to this new class of potent drugs is the additional blocking of low level viral replication by attacking a different target. The integrase inhibitors, in addition to other new drugs that attack different targets such as entry and viral maturation, could possibly be used to create even more potent drug regimens that we use today. The dream of eradication of HIV from the host has resurfaced because of these additional new classes of drugs. Although integrase inhibitors do not penetrate all compartments, the more rapid viral decay that has been observed with their use in treatment-naïve patients suggest that these drugs may favorably impact overall viral burden. Additionally, in a small group of patients, viral DNA was significantly decreased compared to standard therapy. ${ }^{35}$ Future trials are already in progress looking at intensification strategies in patients already suppressed by conventional ART. Another approach is more intensive initial therapy. Where these approaches will lead remains to be seen; however as multiple eradication strategies are appearing on the horizon, it is likely that the integrase inhibitors, with their good tolerance and high potency, are likely to play an important role.

\section{Summary}

Integrase inhibitors are already playing an important role in the armamentarium of antiretroviral therapy; raltegravir has received accelerated marketing approval for use in treatment-experienced patients in the United States and Europe. Further worldwide approvals are expected shortly.
Elvitegravir is progressing to phase III in its development. The combination of high potency in treatment-naïve and experienced patients, good tolerance, favorable pharmacokinetic properties, no significant food effect, no observed fetal and metabolic toxicity profile, makes these compounds a significant addition to the ART regimens available today. The observation that integrase inhibitors positively affect viral dynamics challenges the current understanding of HIV-1 turnover and compartmentalization. The implication for this phenomena in the context of high potency and good tolerability place integrase inhibitors under intense interest at the present time.

\section{Disclosures}

The authors have no conflicts of interest to disclose.

\section{References}

1. World Health Organization and United Nations Programme on HIV/ AIDS. AIDS epidemic update: December 2007. Geneva: World Health Organization; 2007.

2. World Health Organization. Towards universal access: scaling up priority HIV/AIDS interventions in the health care sector; progress report, April 2007. Geneva: World Health Organization; 2007.

3. Cohen J, Lester B. AIDS research. Trials of NIH's AIDS vaccine get a yellow light. Science. 2007;318(5858):1852.

4. Sekaly RP. The failed Merck vaccine study: a step back or a launching point for future vaccine development? J Exp Med. 2008;205(1):63-77.

5. van de Wijgert JH, Shattock RJ. Vaginal microbicides: moving ahead after an unexpected setback. AIDS. 2007;21(18):2369-2376.

6. Trial and failure. Nature. 2007;446(7131):1.

7. The Stategies for Management of Antiretroviral Therapy (SMART) Study Group. CD4+ count-guided interruption of antiretroviral treatment. N Engl J Med. 2006;355:2283-2296.

8. Correll R, Klibanov OM. Integrase inhibitors: a new treatment option for patients with human immunodeficiency virus infection. Pharmacotherapy. 2008;28(1):90-101.

9. Croxtall JD, Lyseng-Williamson KA, and Perry CM. Raltegravir. Drugs. 2008;68(1):131-138.

10. Markowitz M, Morales-Ramirez JO, Nguyen By, Kovacs CM, et al. Antiretroviral activity, pharmacokinetics, and tolerability of MK-0518, a novel inhibitor of HIV-1 integrase, dosed as monotherapy for 10 days in treatment-naïve HIV-1-infected individuals. J Acquir Immune Defic Syndr. 2006;43(5):509-515.

11. Yoder KE, Bushman FD. Repair of gaps in retroviral DNA integration intermediates. J Virol. 2000;74(3):11191-11200.

12. Savarino A. In-silico docking of HIV-1 integrase inhibitors reveals a novel drug type acting on an enzyme/DNA reaction intermediate. Retrovirology. 2007;4:21.

13. Pommier Y, Johnson AA, Marchand C. Integrase inhibitors to treat HIV/AIDS. Nat Rev Drug Discovery. 2005;4:236-248.

14. Savarino A. A historical sketch of the discovery and development of HIV-1 integrase inhibitors. Expert Opin Investig Drug. 2006; 12:1507-1522.

15. Hazuda DJ, Felock P, Witmer M, et al. Inhibitors of strand transfer that prevents integration and inhibit HIV-1 replication in cells. Science. 2000;287:646-650.

16. Anker M, Corales RB. Raltegravir (MK-0518): a novel integrase inhibitor for the treatment of HIV infection. Expert Opin Investig Drugs. 2008;7(1):97-103. 
17. Isentress (raltegravir): US prescribing information. Whitehouse Station, NJ: Merck and Company, Inc; 2007.

18. Iwamoto M, Wenning LA, Petry AS, et al. Safety, tolerability, and pharmacokinetics of raltegravir after single and multiple doses in healthy subjects. Clin Pharmacol. 2008;83(2):293-299.

19. Kassahun K, Mcintosh I, Cui D, et al. Metabolism and disposition in humans of raltegravir (MK-0518), an anti-AIDS drug targeting the HIV-1 integrase enzyme. Drug Metab Dispos. 2007;35(9):1657-1663.

20. Markowitz M, Nguyen BY, Gotuzzo E, et al. Rapid and durable antiretroviral effect of the HIV-1 integrase inhibitor raltegravir as part of combination therapy in treatment-naïve patients with HIV-1 infection: results of a 48-week controlled study. J Acquir Immune Defic Syndr. 2007;46(2):125-133.

21. Mistry G, Wenning L, Petry A, et al. Atazanavir modestly increases plasma levels of MK-0518 [abstract no. MOPEB109]. In: Abstracts of the 4th International AIDS Society Conference on HIV Pathogenesis, Treatment and Prevention; July 22-25, 2007; Sydney. p. 76.

22. Grinsztejn B, Nguyen BY, Katlama C, et al. Safety and efficacy of the HIV-integrase inhibitor raltegravir (MK-0518) in treatment-experienced patients with multidrug-resistant virus: a phase II randomized controlled trial. Lancet. 2007;369:1261-1269.

23. Iwamoto M, Kassahun K, Troyer MD, et al. Lack of pharmacokinetic effect of raltegravir on midazolam: in vitro/in vivo correlation. J Clin Pharmacol. 2008;48(2):209-214.

24. DeJesus E, Berger D, Markowitz M, et al. Antiviral activity, pharmacokinetics, and dose response of the HIV-1 integrase inhibitor GS-9137 (JTK-303) in treatment-naïve and treatment-experienced patients. J Acquir Immune Defic Syndr. 2006;43(1):1-5.

25. Shimura K, Kodama E, Sakajami Y, et al. Broad antiretroviral activity and resistance profile of the novel human immunodeficiency virus integrase inhibitor elvitegravir (JTK-303/GS-9137). $J$ Virol. 2008;82(2):764-774.

26. Cooper DA, Steigbigel RT, Gatell JM, et al. Subgroup and resistance analyses of raltegravir for resistant HIV-1 infection. $N$ Engl J Med. 2008;359:355-365.
27. Steigbigel RT, Cooper DA, Kumar PN, et al. Raltegravir with optimized background therapy for resistant HIV infection. $N$ Engl J Med. 2008;359:339-354.

28. European Medicines Agency. European Public Assessment Report Scientific Discussion, Isentress. 16 November 2007. www.emea.eu/ humandocs/humans/EPAR/isentress/isentress.htm.

29. Zolopa A, Mullen M, Berger D, et al. The HIV integrase inhibitor GS-9137 demonstrates potent antiretroviral activity in treatment-experienced patients. In: Abstracts of the 14th conference on retroviruses and opportunistic infections, Los Angeles, USA, February 25-28, 2007. Abstract 143LB.

30. Roquebert B and Descamps D. La resistance du VIH aux inhibiteurs de l'integrase. Virologie. 2007;11(special): S175-82.

31. Hazuda DJ, Miller MD, Nguyen BY, Zhao J. Resistance to the HIVintegrase inhibitor raltegravir: analysis of protocol 005 , a phase 2 study in patients with triple-class resistance to HIV-1 infection. Antiviral Ther. 2007;12(5):S10. Abstract 8

32. Jones G, Ledford R, Yu F, McColl D. Resistance profile of HIV-1 mutations in vitro selected by the HIV-1 integrase inhibitor, GS-9137 (JTK-303). In: Abstracts of the conference on retroviruses and opportunistic infections, Los Angeles, USA, February 25-28, 2007. Abstract 627.

33. McColl DJ, Fransen S, Gupta S, et al. Resistance and cross-resistance to first generation integrase inhibitors: insights from a phase 2 study of elvitegravir (GS-9137). Antiviral Ther. 2007;12:S11. Abstract 9.

34. Murray JM, Emery S, Kelleher AD, etl al. Antiretroviral therapy with the integrase inhibitor raltegravir alters decay kinetics of HIV, significantly reducing the second phase. AIDS. 2007;21(17):2315-2321.

35. Arponen S, Benito J, Lozano S, et al. More pronounced effect of integrase inhibitor raltegravir on proviral DNA reduction that other antiretroviral drugs in patients achieving undetectable viremia. In: Program Abstracts, 15th Conference on Retroviruses and Opportunistic Infections, February 3-6, 2008. Boston, USA. Abstract 796.

36. Hazuda DJ. Inhibitors of human immunodeficiency virus type I integration. Curr Opin HIV AIDS. 2006;1:212-217. 